\title{
IDENTIFICACIÓN DE LAS DINÁMICAS DE PARTICIPACIÓN LABORAL DE LAS MUJERES QUE HACEN PARTE DEL POLI MEDELLÍN.
}

\author{
Identification of the dynamics of labor participation of women who are part of the Poli \\ Medellín
}

\author{
Marisol Salamanca Olmos \\ msalamanca@poligran.edu.co \\ Nancy Ríos Yepes Gloria \\ griosyep@poligran.edu.co \\ John Edwin Baena Gallego \\ jebaenag@poligran.edu.co \\ Ana María García Villa \\ angarcia25@poligran.edu.co \\ Alejandra Vanesa Gil Rodríguez \\ algilrod@poligran.edu.co
}

Institución Universitaria Politécnico Grancolombiano Programa de Negocios Internacionales

Medellín-Colombia

\begin{abstract}
Resumen
Al identificar cual es el papel de la mujer dentro de la estructura de mercado nos encontramos con la existencia de factores tangibles que explican la brecha educativa, cultural, experiencial e intangibles que tal vez son los que toman mayor fuerza y marcan la desventaja del papel de la mujer en la inserción económica y actualmente se comprenden a través de la brecha oscura formada a partir de estereotipos como el machismo, prejuicios y la maternidad, que analizados todos en conjunto pueden llegar a excluirla del funcionamiento de la estructura económica, y se convierten en paredes de cristal que difícilmente se pueden atravesar, en este sentido, se hace necesario entender su funcionamiento. La problemática se plantea a partir de ¿cuáles son las dinámicas de la participación laboral de las mujeres que hacen parte del Poli Medellín?, identificando la manera de lograr caracterizar los diferentes roles que desempeña la mujer desde ser profesionales, empleadas, estudiantes, realizar labores en el hogar y así poder reconocer la existencia de brechas salariales, discriminación, identificación de roles y demás elementos que nos arroje la investigación.

La metodología por utilizar en esta investigación parte del enfoque mixto en el cual se utilizan técnicas tanto cualitativas como cuantitativas, lo que permite realizar un análisis de las dinámicas sociales y económicas de las mujeres del politécnico además de su participación laboral

En este sentido, se hace necesario entender su funcionamiento y se deben dar pasos hacia la transformación de las desigualdades y el fin de prejuicios, los roles de género y los estereotipos que tanto daño generan porque sólo con hombres y mujeres conviviendo en igualdad será posible solucionar los conflictos políticos, la prevención de las enfermedades, el crecimiento, desarrollo y, en definitiva, garantizar la sostenibilidad económica.
\end{abstract}

Palabras Clave: Brecha salarial, estereotipos, independencia económica, desigualdad de género. 
Introducción

La mujer ha luchado por ser incluida y aceptada en dinámicas laborales y de decisión ejecutiva, no obstante, luego de terminar su jornada laboral, continúa con labores de ama de casa, cuidado de hijos, esposa, entre otras, sin ser valorada, compensada, ni reconocida. Esta investigación pretende reconocer el papel de la mujer en la dinámica social y económica de los trabajadores y estudiantes del Politécnico Grancolombiano, sede Medellín, además de cuantificar su aporte a la economía en el ámbito empresarial.

Desde esta óptica es importante entender cuáles son los diferentes roles que desarrolla la mujer desde el hogar, su profesión, el trabajo, la independencia económica, la educación, labores que en algunos casos sugieren poca valoración económica, escasos espacios donde desarrollarse, una lucha continua para lograr conciliar el tiempo dedicado a su desarrollo profesional, y adicionalmente cumplir sus labores en el hogar.

Elementos que en conjunto acentúan esa brecha entre hombres y mujeres, y analizado específicamente desde la óptica colombiana, de acuerdo con cifras del DANE la tasa de desempleo en Colombia en el 2019 fue de 12,8\%, que corresponde a 9.8\% de hombres desocupados respecto a $16.9 \%$ para las mujeres, lo que representa una brecha de género de 7.1\%. Situación preocupante si se considera desde la perspectiva de crecimiento y desarrollo para la economía colombiana que ha venido recuperándose lentamente desde el 2015, pero que resulta aún más discriminatoria para las mujeres, quienes se ven enfrentadas a romper con esas paredes de cristal desde su condición de raza, estrato, nivel académico.

En el ámbito empresarial son aún más difíciles las condiciones, pues las mujeres se enfrentan a las diferencias marcadas versus los hombres, como la existencia de una brecha de

ingreso que promedio para Colombia es del 17.6\% (Departamento Nacional de Planeación, 2019) y la constante de ver como son más los hombres que ocupan cargos directivos y las mujeres terminan encasillando dentro de cargos asistenciales ya sea por falta de confianza en la capacidad de desempeño profesional o por las condiciones que le significan al empleador la contratación de una mujer, esto pensado en términos de productividad al relacionar el embarazo, la licencia de maternidad, permisos para asistir a citas médicas, o cuidado de los hijos entre otros.

Desde el Estado, el gobierno en su Plan Nacional de desarrollo para el 2018 - 2022 propone lo que ha denominado "Pacto de equidad para las mujeres", con objetivos dirigidos a promover la participación de las mujeres en el mercado laboral, promover sus derechos y participación del 50\% en cargos públicos, reducción de la brecha de ingreso mensual y la contratación de 880.000 empleos para mujeres (Departamento Nacional de Planeación, 2019), vale la pena resaltar que el 50\% de su gabinete está conformado por mujeres adicional a la vicepresidenta que es la primera mujer en ocupar este cargo, formadas en las áreas de derecho, economía, administración de empresas, ingeniería y ciencia política (Guevara Benavides, 2018).

En la actualidad la mujer se presenta multifacética, realizando diferentes tareas en periodos cortos de tiempo con el fin de cumplir con los distintos roles que desempeña enmarcados dentro de lo social, político y económico en el afán de buscar reconocimiento, valoración y compensación económica, evidenciándose que no es suficiente con que sean profesionales, empleadas o estudiantes, sino que además culminen su día empatando con una nueva jornada laboral que no es remunerada ni cuantificada.

Es por ello que se hace pertinente resaltar y visibilizar el papel estratégico y aporte a la economía que juega la mujer, desde los distintos roles sociales que desempeña (madre, esposa, trabajadora, empresaria y estudiante) y sobre todo, resaltar su funcionalidad en las dinámicas empresariales y 
organizacionales, además de generar espacios de prácticas donde los y las estudiantes de las áreas administrativas (negocios, contaduría y administración de empresas) apliquen conocimientos y desarrollen competencias laborales que les exige la dinámica del mercado, además de permitirles afianzar conocimientos y ganar confianza para enfrentarse al mundo laboral y profesional.

Este proyecto permite identificar cual es la perspectiva local de la mujer, en términos de la satisfacción laboral y personal de la mujer Poligrancolombiana, entendiendo su participación, valoración y la conciliación del tiempo entre las actividades que desarrolla en el campo laboral que para ella le significan alcanzar el éxito profesional y aquellas actividades enmarcadas dentro del marco legal del cuidado de la mujer.

Trabajar este tema de las dinámicas de la participación laboral de la mujer en nuestra sociedad, implica hacer un análisis retrospectivo del contexto social impregnado en la desigualdad de género con normas y pautas de comportamientos rígidas, que han marcado diferencias entre hombres y mujeres, las cuales han sido influenciadas y acentuadas desde las prácticas tradicionales de las distintas culturas, esto, desde sociedades primitivas hasta el imperio capitalista actual.

Esta situación social y visiblemente marcada aún más en nuestros días, ha llegado a convertirse en motivos de preocupación para gobiernos de países del mundo, pasando a legislarse en torno a ella en el propósito de reconocer y equiparar socialmente derechos vulnerados del sexo femenino desde la antigüedad, no menos sin reconocer, la lucha encarnada que han tenido que dar las mujeres del mundo a través de distintos movimientos feministas conformados y la tenacidad de mujeres lideresas auto determinadas, en la tarea de exigir a estos gobiernos el reconocimiento de derechos, igualdad e inclusión social y la no discriminación en aspectos religiosos, raciales, sexuales, de genero entre otros.

\section{Marco Teórico}

En una economía y en su estructura de mercado, dentro del papel que desempeña cada individuo surge el interrogante cuál es el espacio que desempeña la mujer, no solo para que esta satisfaga sus necesidades básicas, sino que encuentre dentro de la sociedad un espacio para desarrollarse, crecer, educarse, trabajar y a su vez desempeñar su papel como madre, cabeza de hogar, o sencillamente aquella mujer que desea también alcanzar cargos directivos, políticos, poder tener un espacio dentro de la sociedad, sin que exista discriminación.

En ese sentido es importante mencionar la participación de las mujeres de acuerdo con el informe (ONU MUJERES, 2019): En febrero de 2019, solo 3 países tenían un 50 \% o más de mujeres en el Parlamento, ya sea en la cámara única o baja: Ruanda con el $61,3 \%$, Cuba con el $53.2 \%$ y Bolivia con el $53,1 \%$. No obstante, un número más elevado de países ha alcanzado el $30 \%$ o más de representación femenina", de manera que lleva a entender que la mujer ha venido poco a poco posicionándose en diferentes escenarios.

En el caso Colombiano el gobierno en su Plan Nacional de desarrollo para el 2018 - 2022 propone lo que ha denominado "Pacto de equidad para las mujeres", con objetivos dirigidos a promover la participación de las mujeres en el mercado laboral, promover sus derechos y participación del $50 \%$ en cargos públicos, reducción de la brecha de ingreso mensual y la contratación de 880.000 empleos para mujeres (Departamento Nacional de Planeación, 2019), vale la pena resaltar que el 50\% de su gabinete está conformado por mujeres adicional a la vicepresidenta que es la primera mujer en ocupar este cargo, mujeres formadas en derecho, economía, administración de empresas, ingeniería y ciencia política (Guevara Benavides, 2018).

Con la globalización se ha podido dar un gran salto, según el estudio sobre Éxito y satisfacción laboral y personal cómo lo perciben mujeres que trabajan en Bogotá, Colombia, realizado por (Carlier \& 
Towsend, 2014), afirman: "Hablar de éxito y satisfacción laboral en mujeres es forzosamente algo nuevo, ya que su ingreso masivo en el campo laboral remunerado se produjo a partir de la segunda mitad del siglo XX".

Estos avances que ha dado la mujer en el terreno de lo público, lo privado, lo productivo ha implicado una inserción en el sistema, esto favorecido por la evolución que ha tenido la sociedad, la globalización, así lo confirman los autores del libro Mujeres y Economía:

La globalización, junto con los adelantos en el terreno de la informática han permitido que cada vez sea mayor la comunicación entre las mujeres de todo el mundo como lo atestiguan las conferencias nacionales e internacionales. La participación de las mujeres es creciente, pero no óptima; aún en muchos países se las margina, sin considerar que son agentes de cambio a través de la reproducción de la fuerza de trabajo y de los valores sociales.(Arroyo et al., 2002).

De acuerdo con lo anterior se demuestra una vez más que las mujeres dedican más tiempo que los hombres a sus labores domésticas, sobre ella recae las responsabilidades familiares, lo que le puede impedir acceder a otras oportunidades laborales.

Es así como encontramos textos con una visión de valorar la mujer, como es el caso del libro "Quien le hacía la cena a Adam Smith" donde Marcal Katrine, expone:

Así como hay un segundo sexo hay una segunda economía. El trabajo que tradicionalmente han hecho los hombres es el que cuenta, el que define el panorama económico mundial. El trabajo de la mujer es el que va en segundo lugar, lo otro: todas las labores que él no desempeña, pero de las que, al mismo tiempo, depende para poder realizar sus propias tareas. (Marçal, 2016).

Entender entonces el funcionamiento de la actual estructura económica capitalista, también llamado sistema económico mixto y cómo esta estructura es vinculante a la mujer, permitirá conocer que se está haciendo para disminuir esa brecha y como desde la academia y los espacios de la administración local, regional y nacional se puede empoderar y visibilizar a la mujer dentro de la sociedad.

En el Documento de Trabajo, 191. Serie Programa Nuevas Trenzas, 15, afirma, Economía del Cuidado, presentan:

En primer lugar, las mujeres en todos los países trabajan más horas en total que los hombres, especialmente en Uruguay y México. En segundo lugar, las mujeres tienden a trabajar más horas en actividades no remuneradas, mientras que los hombres se dedican más a actividades remuneradas. En tercer lugar, las mujeres rurales dedican muchas más horas al trabajo en general y en especial a trabajos no remunerados, tanto en comparación con los hombres como con las mujeres urbanas. En Colombia, las mujeres rurales trabajan en total 64 horas semanales. (Peña \& Uribe, 2013).

No solo queremos mostrar las limitaciones que dificultan la incursión de la mujer en las actividades empresariales y dinámica económica regional, sino visibilizar su impacto a nivel emocional y como una mujer, o una persona valorada, reconocida puede dar un mayor aporte a la sociedad, es así como en estas dinámicas que desarrolla la mujer, se encuentra según el documento que realiza el análisis comparativo de mujeres con trabajo remunerado y amas de casa. Clínica y Salud. 2000;11(2):195-229.: "Una gran parte de los estudios realizados en Estados Unidos y en el mundo occidental muestran que en conjunto las mujeres que trabajan fuera de casa poseen mejor salud mental que las que se dedican a las labores domésticas" (Escalera Izquierdo \& Sebastián Herránz, 2000).

Desde varias ópticas es importante entender cuáles son los diferentes roles que desarrolla la mujer desde el hogar, su profesión, la independencia económica, la educación, labores que algunos casos sugieren poca valoración económica, escasos espacios donde desarrollarse y una lucha continua para 
lograr conciliar el tiempo dedicado a su desarrollo profesional y adicionalmente el cumplimiento de muchas de labores en el hogar.

Sin pretender adelantarnos en los resultados de esta investigación, varios estudios, arrojan un resultado muy positivo desde que la mujer incursionó en el mercado laboral, su aporte a la economía y a la sociedad es muy valioso, pero aún falta ese reconocimiento, es así como los autores del libro Mujeres y trabajo: "cambios impostergables", presentan:

Numerosas investigaciones dan cuenta de la masiva incorporación de las mujeres al trabajo remunerado y los efectos positivos que esto ha tenido en cuanto al desarrollo de la autonomía, realización personal y posibilidades de organización. Pero también se ha observado que la inclusión de las mujeres en la ciudadanía no se ha correspondido con la posesión de los beneficios de la ciudadanía social vinculados al derecho al trabajo"(León T, 2003).

Es importante entender el reconocimiento que tiene la mujer dentro del actual sistema económico, es así como, Carrario Martha, en su obra "Los retos de las mujeres en tiempo presente" expone: En el caso del modelo económico capitalista es posible que la falta de reconocimiento no sea al propio trabajo doméstico, como a la conexión que tiene con la propia producción capitalista y con ello una negación de los costes de la reproducción de la fuerza de trabajo. ¿Sería posible mantener el capitalismo neoliberal, y la ganancia que genera la fuerza de trabajo asalariado si la reproducción de la fuerza de trabajo que se realiza en la esfera doméstica debiera ser asalariada también, o directamente fuera abandonada?(Carrario, 2008).

Es en esta perspectiva entonces se sugería que el sistema capitalista como tal, en lugar de ser un factor de tendencia que facilite la participación y reconocimiento activo de la mujer en las dinámicas sociales y económicas, se comporte como una ruptura que conlleve a la desaceleración de los espacios y participación que han ganado las mujeres, adicional se puede inferir que se está lejos de poder tener un reconocimiento en términos de ingreso por el trabajo doméstico que realizan las mujeres, y que es el mismo sistema capitalista no incluye dentro de sus actividades productivas estas labores desempeñadas en su mayoría por mujeres. Carosio Alba en la Revista Venezolana de estudios de la mujer señala:

En muchos países, cualquiera que sea su nivel de desarrollo, el acceso al mercado del trabajo es sistemáticamente más difícil para las mujeres que para los hombres. Con frecuencia se niegan a las mujeres las posibilidades de ejercer un empleo asalariado a causa de sus responsabilidades familiares, de su falta de calificaciones, de barreras sociales y culturales o por falta de puestos de trabajo. En este contexto, el ejercicio de una actividad independiente o la creación de una empresa -por lo general una microempresa- son con frecuencia los únicos medios de que disponen las mujeres para obtener unos ingresos que les permitan garantizar su subsistencia y la de sus hijos.(Carosio, 2004).

Para el corte del 2014 -2017, los emprendimientos femeninos aumentaron un 68\% pasando del $15 \%$ al $25 \%$ respectivamente, posicionando a Colombia como el país que ha venido logrando aumentos importantes en emprendimiento a nivel Latinoamérica y el Caribe, en este sentido los estudios del Global Entrepreneurship Monitor (GEM), señala que el emprendimiento liderado por las mujeres ha venido en ascenso. La investigación con corte a 2018 muestra, el indicador Actividad Empresarial Total (TEA), colocando a Colombia dentro de Latinoamérica en el cuarto lugar después de Guatemala (27,5\%), Chile (25,1\%) y Perú (22,4\%) (Global Entrepreneurship Monitor, 2019).

Según el Estudio de la Actividad Empresarial (Global Entrepreneurship Monitor, 2019) las personas que emprenden se encuentran en un rango de edad de los 25 y 34 siendo mayor la proporción de hombres respecto a las mujeres y en el rango de edad de 45 a 54 años, son las mujeres las que emprenden en una mayor proporción con un 10.1\%. En relación con los emprendimientos de las mujeres para el año 
2018 disminuyó un 0,4\% esto en contraste con los emprendimientos de los hombres que se incrementó en un $5.7 \%$, incrementando la brecha de género en un7.1\% respecto al año anterior 2017 donde la brecha era del $1 \%$, la TEA de las mujeres se encuentra en el $17.8 \%$ respecto a la de los hombres de $24.9 \%$.

Las mujeres emprenden motivadas por la flexibilidad que le puede significar ser independientes y los hombres lo hacen por la necesidad de emplearse, esto según estudio de la Asociación de Emprendedores de Colombia y Asela (Portafolio, 2019), adicional vale la pena mencionar que las mujeres tienen la motivación de poder tener un control sobre el tiempo que puede repartir entre su emprendimiento y el tiempo dedicado a la familia y la posibilidad de poder mejorar sus ingresos.

Si se quiere avanzar, y reconocer el trabajo de la mujer, se deben formular diferentes alternativas para visibilizar y darle un valor al trabajo del hogar no remunerado, es así como León, Irene en Mujeres en resistencia:

Tanto el universo social como el doméstico, incluyendo cuestiones tan íntimas como la concepción, están cada vez más penetrados por el neoliberalismo, de allí que para formular alternativas económicas feministas es preciso analizar la articulación entre el trabajo doméstico y el asalariado y poner en evidencia algunas transformaciones técnico- científicas, sociales y económicas, para desarrollar una teoría que supere en gran medida

lo que se ha hecho hasta ahora con el trabajo doméstico. En otras palabras, es preciso cristalizar una propuesta encaminada a ecologizar el mundo, lo que incluye la casa. (León Ed., 2005).

Ya hay elementos claros, hay que transformar el modelo económico actual si es el caso, el trabajo doméstico como lo menciona León Irene constituye el punto de apoyo invisible, se debe articular el tiempo de trabajo, el de salario y visibilizarlo, remunerarlo y a su vez visibilizar desde una perspectiva de género de manera que tanto hombres y mujeres puedan acceder en igualdad de condiciones, derechos y ser valorados dentro de la estructura económica de la sociedad.

Dentro de los elementos que se espera evidenciar como resultado de la investigación es la existencia de los techos de cristal que se convierten en barreras de acceso para las mujeres y se convierten en elementos que dificultan su acceso a puestos de responsabilidad o mayor jerarquía. acuerdo como lo plantea la Revista Apuntes de Psicología:

A pesar de que se han integrado en el mercado de trabajo de forma masiva en las últimas décadas, su posición es de clara desventaja con respecto a la de sus compañeros. Esta desigualdad se ve reflejada en su dificultad para acceder a puestos de poder, debido a la existencia de una última barrera, denominada techo de cristal, basada en prejuicios hacia las mujeres, que les dificulta y/o impide llegar a la cima.(Agut Nieto \& Martín Hernández, 2007).

Al consultar diferentes textos sobre el papel de la mujer en la sociedad, se encuentran autoras, que piden resolver el problema de discriminación y de darle un papel protagónico a la mujer en la sociedad, es así como Virginia Wolf, en su obra una Habit Acción Propia, plantea:

Cuanto podía ofreceros era una opinión sobre un punto sin demasiada importancia: que una mujer debe tener dinero y una habitación propia para poder escribir novelas; y esto, como veis, deja sin resolver el gran problema de la verdadera naturaleza de la mujer y la verdadera naturaleza de la novela (Wolf, 1967).

De esta manera nos deja ver que siempre la mujer ha venido reclamando ese reconocimiento dentro de la sociedad, pero más allá, es poder ser valorada para poder tener participación y que tanto hombres como mujeres pueden tener las mismas oportunidades y ejercer en igualdad de condiciones los diferentes roles. 
Método

La metodología por utilizar en esta investigación parte del enfoque mixto en el cual se utilizan técnicas tanto cualitativas como cuantitativas, lo que permite realizar un análisis de las dinámicas sociales y económicas de las mujeres del politécnico además de su participación laboral, dentro de la muestra a trabajar no se excluyen a los hombres pues se requiere su mirada y concientización sobre las labores que realizan las mujeres.

La investigación cuantitativa la evaluaremos a través de una encuesta virtual y presencial de aproximadamente 26 preguntas alrededor de los temas de educación, brecha salarial, uso del tiempo y rol en el hogar, la muestra se seleccionará bajo un nivel de confianza del $90 \%$ y un error del $5 \%$, aplicándoseles a los y las estudiantes, docentes y colaboradores del Politécnico Grancolombiano Sede Medellín (Ver la encuesta en el anexo 1) durante dos semanas, preferiblemente entre la segunda y tercera semana del mes de noviembre de 2019.

Respecto a la investigación cualitativa se realizará de tipo etnográfico, porque permite un análisis en el que se observa lo que dicen, hacen, actúan y piensan sus actores y protagonistas, y a su vez posee un rigor teórico, técnico y metodológico con principio y flexibilidad para el registro y análisis de la información por ello utilizaremos las técnicas de grupo focal y entrevistas que nos permitirán una mayor profundidad en las respuestas de carácter cuantitativo estas actividades se realizaran durante el primer semestre del año 2020.

El procedimiento por seguir se divide en tres etapas:

Etapa I: Exploración: se hace una revisión documental, se construye el marco teórico y se inicia un acercamiento con la comunidad del politécnico a través de una encuesta que se detalla en el anexo 1. Etapa II. Análisis: se realiza una contextualización de los resultados de la encuesta, se profundiza a través de 4 grupos focales donde se avanzan en las relaciones socioeconómicas, uso del tiempo, roles en el hogar, educación y brecha, se procede a la organización de información.

Etapa III. Interpretación: se interpretan los resultados obtenidos en las etapas anteriores, y se procede con la redacción del artículo.

\section{Estructura Metodológica}

Exploración

Análisis de Campo

Interpretación y Entrega de Resultados

- Diseño y evaluación de Instrumentos.

- Redefinición de categorías de estudio de acuerdo con los objetivos específicos.

- Redacción del artículo

- Ejecución de técnicas de investigación.

- Comparativos de resultados de otros estudios desde el Dane o Internos.

- Participación como ponentes en los diferentes encuentros de investigación de la ciudad o el país.

- Evaluación de los instrumentos.

- Análisis e interpretación de resultados

- Puesta en marcha y revaluación en caso de solicitarse 


\section{Resultados Esperados}

La investigación pretende identificar la manera de lograr caracterizar los diferentes roles que desempeña la mujer que hace parte del Poli Medellín y las percepciones de los hombres frente al papel de la mujer, los cuales van desde ser profesionales, empleadas, estudiantes, realizar labores en el hogar, etc, y así poder reconocer la existencia de brechas salariales, discriminación, identificación de roles y demás elementos que nos arroje la investigación.

La gran mayoría de mujeres que hacen parte del Poli sede Medellín son personas en edad productiva y que además por sus diferentes obligaciones estudian en la jornada nocturna, lo que "les facilita" realizar sus diferentes labores durante el día pero que también las obliga a renunciar a su tiempo de descanso para formarse e incluso tener la sensación permanente de que el tiempo no es suficiente para cumplir con las actividades.

Durante el desarrollo de la encuesta, las personas evaluadas tendrán diferentes sensaciones sobre la distribución del tiempo, las múltiples actividades que se llevan a cabo en el hogar como lo es el cuidado de los seres queridos sin ninguna recompensa monetaria, la vida social y el tiempo dedicado a su desarrollo personal.

Para ello se analizara las respuestas de los instrumentos utilizados, partiendo de la claridad del público objetivo, tomando como base una muestra significativa, con el fin de lograr respuestas representativas de la población, con los cuales esperamos alcanzar cada uno los objetivos planteados, tales como conocer el papel de la mujer en la dinámica social, económica y regional del Politécnico Grancolombiano, sede Medellín, identificar las limitaciones que dificultan la incursión de la mujer en las actividades empresariales, potenciar procesos de autogestión en el juego de los diferentes roles sociales que ha asumido la mujer, principalmente su incursión en la vida laboral con remuneración económica.

\section{Discusión y Conclusiones:}

Las mujeres se han visto obstaculizadas en los puestos de trabajos, más que todo por temas relacionados a la maternidad y las dudas que surgen en la sociedad sobre la capacidad para realizar las funciones de su trabajo, esto conlleva al surgimiento de temas que se relacionan a difíciles situaciones, las cuales al pasar del tiempo las mujeres han tenido que enfrentar, entre estas la brecha salarial.

Debido a esto gran parte de la población femenina se limita a realizar actividades que puedan ser cuestionadas por la sociedad, es decir, no encuentran un espacio en donde puedan desarrollarse, dejarlas ser mujeres empoderadas con grandes habilidades para romper cualquier barrera y no solo se desempeñen en actividades caseras, hoy en día existen personas que son conscientes del empoderamiento y la capacidad que una mujer puede llegar a tener, hace falta transformar el pensamiento de gran parte de la población mundial, para dejar a un lado el machismo y darle inicio a una sociedad más equitativa, aceptando las diferencias de género y dándole oportunidad a la capacidad femenina.

Un factor que muestra las desigualdades existentes entre géneros se relaciona con el aprovechamiento de los tiempos y el hecho de que, existen aún ciertas actividades que siguen considerándose esencialmente femeninas como las relacionadas con el hogar y la familia. A pesar de que la mujer se ha incorporado al mundo laboral, no ha dejado de atender las labores de la casa, de modo que ha tenido que sacrificar otras actividades, como la propia promoción en el trabajo, su recreación, cuidado de sí misma, entre otras. 
Sin duda alguna la revisión teórica de los diferentes autores, la retrospectiva de la participación de abuelas, madres y la mujer actual que se está caracterizando, permiten identificar un escenario más positivo, donde la mujer ha logrado una mayor independencia, acceso a la educación, participación en altos puestos ejecutivos, cargos políticos, independencia económica, decisión sobre su cuerpo, decisiones de ser madres, legislación que en cierta medida garantiza los derechos de las mujeres y terminan otorgándole un reconocimiento en la sociedad y un escenario prospectivo promisorio, por lo cual sin duda alguna esta dinámica de participación de la mujer en la sociedad continuara en ascenso.

De esta manera, no sólo es cuestión de conceder y reconocer una serie de derechos o de mejorar ciertas condiciones de vida relacionadas con la salud, la vivienda o la educación, sino de que las mujeres conquisten espacios que le eran vetados, tomando decisiones estratégicas que antes eran exclusivamente de los hombres. En la medida en que se puedan identificar estos espacios, se podrá afirmar que se ha avanzado en materia de equidad y como sociedad.

La diversidad de género permite mejorar el rendimiento de las empresas, la experiencia y las diversas investigaciones de ONU Mujeres, Pacto Global, agencias de Naciones Unidas, el Banco Mundial y el Foro Económico Mundial refuerzan estos hallazgos. Los gobiernos reconocen que la inclusión de la mujer lleva al desarrollo y que alcanzar los Objetivos de Desarrollo Sostenible y los planes nacionales de desarrollo depende de la equidad de género.

Una vez se reconoce el verdadero papel que la mujer juega en la sociedad, a través de discusiones teóricas, investigaciones, estudios, documentación, se aportan elementos que muestran el impacto que tendrá la economía con su contribución como actora protagónica, lo cual permite avanzar en la vida social, cultural, económica.

Es importante poder validar la demanda de trabajo de cuidado actividades que recaen sobre las mujeres ya que por lo general son ellas quienes cuidan a los niños, a los enfermos, ancianos y es un trabajo no remunerado que no refleja la carga total del trabajo social que da cuenta del papel fundamental que tiene este en la economía.

La revisión bibliográfica permitió contrastar y validar en una primera fase de la investigación la importancia de identificar para la población del Politécnico Grancolombiano sede Medellín, la participación que tienen las mujeres y los hombres dentro de las diferentes actividades económicas, su nivel de satisfacción laboral y personal y hacer un análisis del tiempo que dedican a las actividades del cuidado del hogar, labores domésticas, académicas, personales.

De igual manera resulta importante identificar la existencia de techos de cristal para las mujeres en tiempos en los que se habla de igualdad de género y donde se ha venido reconociendo su aporte que estas hacen a la economía, y que fenómenos como la globalización han sido un medio que facilite su inserción al mercado facilitando los espacios para educarse, tener mayor participación laboral, reconocimiento de sus derechos e identificar las limitaciones que puede tener para desempeñar los diferentes roles.

Identificar las dinámicas de participación laboral de las mujeres en la sociedad es empoderarlas y hacerlas partícipes de la vida económica y en todos los sectores para: Construir economías fuertes, establecer sociedades más justas y estables, alcanzar objetivos acordados internacionalmente sobre desarrollo, sostenibilidad y derechos humanos, mejorar la calidad de vida de las mujeres, hombres, familias y comunidades, e impulsar las operaciones y metas empresariales, es todo un proceso de adentro hacia afuera, se inicia en el ámbito personal y se expande hacia una dimensión colectiva.

Hay muchas razones para empoderar a las mujeres, al igual que hay muchas maneras de contribuir, a un mundo equitativo y sostenible, lo importante es no quedarse de brazos cruzados. Ya lo dijo la escritora y activista política norteamericana Hellen Keller: "No soy la única, pero aun así soy alguien. 
No puedo hacer todo, pero aun así puedo hacer algo. $\mathrm{Y}$ justo porque no puedo hacer todo, no renunciaré a hacer lo que sí puedo" (Ruiz Ruiz, 2015).

\section{Referencias Bibliográficas}

Agut Nieto, S., \& Martín Hernández, P. (2007). Factores que dificultan el acceso de las mujeres a puestos de responsabilidad: una revisión teórica. Apuntes de Psicología, 25(2), 201-214. Retrieved from http://copao.cop.es/files/contenidos/VOL25_2 7.pdf

Arroyo, A., Correa, E., Chaves Hoyos, M., Giron, A., Gonzalez Marin, M. L., Perez Licona, P., \& Rodriguez, P. (2002). Mujeres y Economía (Primera edición). Retrieved from https://ebookcentral-proquest-com.loginbiblio.poligran.edu.co

Carlier, S. I., \& Towsend, P. L. (2014). Éxito y satisfacción laboral y personal: cómo lo perciben mujeres que trabajan en Bogotá (Colombia). Revista Científica Pensamiento y Gestión, (¿Cómo perciben el éxito y la satisfacción laboral, personal y familiar 213 mujeres que trabajan en Bogotá y qué papel juegan los distintos elementos del ámbito familiar y de las condiciones laborales en esa percepción?), 153-181. Retrieved from https://doi.org/10.14482/pege.36.5569

Carosio, A. (2004). Las mujeres y la opción emprendedora: consideraciones sobre la gestión.

Revista Venezolana de Estudios de La Mujer, ProQuest Ebook Central. , 9. Nro 23. Retrieved from https://ebookcentral-proquestcom.loginbiblio.poligran.edu.co/lib/bibliopoligransp/detail.action?docID=3175512

Carrario, M. (2008). Los retos de las mujeres en tiempo presente, ¿Cómo conciliar la vida laboral y la vida familiar? (Volumen XII, pp. 161-173). Volumen XII, pp. 161-173. Retrieved from https://docplayer.es/15214277-Los-retos-de-las-mujeres-entiempo- presente-como-conciliar-la-vida-laboral-y-la-vida-familiar.html

D’Alessandro, M. (2018). Economía Feminista: Las mujeres, el trabajo y el amor. (Segunda Ed).

Argentina: Ediciones B.

Departamento Nacional de Planeación. (2019). Equidad para las mujeres. Retrieved May 16, 2019, from Pactos Transversales website: https://www.dnp.gov.co/DNPN/Plan-Nacional-de-Desarrollo/Paginas/Pactos-Transversales/Pacto-de-equidadpara-las-mujeres/Equidad- para-mujeres.aspx

Escalera Izquierdo, M. E., \& Sebastián Herránz, J. (2000). Trabajo y salud en la mujer: análisis comparativo de mujeres con trabajo remunerado y amas de casa. Clinica y Salud, 11, 195-229. Retrieved from http://search.ebscohost.com/login.aspx?direct=true\&db=aph\&AN=26306812\&site=ehost- live.

Global Entrepreneurship Monitor. (2019). GEM Reportes de Colombia. Retrieved from https://www.gemconsortium.org/economy-profiles/colombia

Guevara Benavides, L. M. (2018, August 8). Quiénes son las mujeres que acompañarán a Iván Duque en el nuevo Gobierno. La Republica. Retrieved from https://www.larepublica.co/especiales/duquepresidente/las-mujeres-que-acompanaran-aivan-duque-en-el-nuevo-gobierno-2757200

León Ed., I. (2005). Mujeres en resistencia. Experiencias, visiones y propuestas (Primera Edición). Retrieved from http://www.bantaba.ehu.es/obs/files/view/Mujeres_en_resistencia.pdf?revision_id=66438\& package_id=66423

León T, M. (2003). Mujeres y trabajo: cambios impostergables. Retrieved from http://bibliotecavirtual.clacso.org.ar/clacso/gt/20101012020005/leon.pdf

Marçal, K. (2016). ¿Quién le hacía la cena a Adam Smith?. Una historia de las mujeres y la economía. Retrieved from 978-84-9992598-1

ONU MUJERES. (2019). Hechos y cifras: liderazgo y participación política. Retrieved from www.unwomen.org/es/what-wedo/leadership-and-political-participation/facts-and-figures

Peña, X., \& Uribe, C. (2013). Economía del cuidado: valoración y visibilización del trabajo no remunerado. CEDE, 34. Retrieved from https://ebookcentral-proquest- com.loginbiblio.poligran.edu.co

Ruiz Ruiz, I. (2015). Hellen Keller “Mujeres.” Retrieved from https://www.behance.net/gallery/28735245/Hellen-Keller-Mujeres Wolf, V. (1967). Una Habitación Propia (Primera edición). Retrieved from http://biblioz.url.edu.gt/Libros/wilde/habitacion.pdf 